VOL. 60 (1999) [197-205]

\title{
ON GROUPS WITH SMALL ORDERS OF ELEMENTS
}

\author{
Narain D. Gupta and Victor D. Mazurov
}

To Bernhard Neumann on his 90 th birthday

For a periodic group $G$, denote by $\omega(G)$ the set of orders of elements in $G$. We prove that if $\omega(G)$ is a proper subset of the set $\{1,2,3,4,5\}$ then either $G$ is locally finite or $G$ contains a nilpotent normal subgroup $N$ such that $G / N$ is a 5-group.

Let $G$ be a periodic group. Denote by $\omega(G)$ the set of orders of elements in $G$. It is obvious that a group with $\omega(G)=\{1,2\}$ is elementary Abelian. Levi and van der Waerden [5] proved that if $\omega(G)=\{1,3\}$ then $G$ is nilpotent of class at most 3. B.H. Neumann [6] described the groups with $\omega(G)=\{1,2,3\}$. Sanov [8] and M. Hall [1] stated that a group $G$ with $\omega(G) \subseteq\{1,2,3,4\}$, respectively with $\omega(G) \subseteq\{1,2,3,6\}$, is locally finite. Nothing is known about local finiteness of groups of exponent 5 , but it follows from [11] that every group $G$ with $\omega(G)=\{1,2,3,5\}$ is isomorphic to the alternating group $A_{5}$.

In this direction, we prove the following results.

THEOREM 1. Let $G$ be a group with $\omega(G)=\{1,3,5\}$. Then one of the following holds:

(i) $G=F T$ where $F$ is a normal 5-subgroup which is nilpotent of class at most 2 and $|T|=3$;

(ii) $G$ contains a normal 3-subgroup $T$ which is nilpotent of class at most 3 such that $G / T$ is a 5 -group.

THEOREM 2. If $\omega(G)=\{1,2,5\}$ then $G$ contains either an elementary Abelian 5-subgroup of index 2, or an elementary abelian normal Sylow 2-subgroup.

THEOREM 3. If $\omega(G)=\{1,2,4,5\}$ then one of the following holds:

(i) $G=T D$ where $T$ is a non-trivial elementary Abelian 2-group and $D$ is a non-Abelian group of order 10;

(ii) $G=F T$ where $F$ is an elementary Abelian normal 5-subgroup and $T$ is isomorphic to a subgroup of a quaternion group of order 8 .

Received 21st April, 1999

This work was carried out during a visit of the second author to the University of Manitoba, Canada.

Copyright Clearance Centre, Inc. Serial-fee code: 0004-9727/99 \$A2.00+0.00. 
(iii) $G$ contains a normal 2-subgroup $T$ which is nilpotent of class at most 6 such that $G / T$ is a 5-group.

In a forthcoming paper, we prove that every group $G$ with $\omega(G)=\{1,2,3,4,5\}$ is locally finite. In this connection, we propose a conjecture that every group in the conclusion of Theorems $1-3$ is also locally finite. This is equivalent to the following

CONJECTURE 1. Let $A$ be an automorphism group of an elementary Abelian $\{2,3\}$-group $G$ such that every non-trivial element of $A$ fixes in $G$ only the trivial element. If $A$ is of exponent 5 then $A$ is cyclic.

\section{NOTATION AND PRELIMINARY RESULTS}

If $H$ is a subgroup of a group $G, x, y \in G, X, Y$ are subsets of $G$ then $x^{y}=$ $y^{-1} x y, X^{y}=\left\{y^{-1} x y \mid x \in X\right\},[x, y]=x^{-1} x^{y}, x^{Y}=\left\{x^{y} \mid y \in Y\right\}, X^{Y}=\left\{x^{y} \mid x \in\right.$ $X, y \in Y\}, N_{H}(X)=\left\{g \in H \mid X^{g}=X\right\},\langle X\rangle$ is the subgroup generated by $X,[X, Y]=$ $\langle[x, y]| x \in X, y \in Y]\rangle, C_{H}(X)=\{h \in H \mid(\forall x \in X)[h, x]=1\}, Z(G)=C_{G}(G)$. For a prime $p, O_{p}(G)$ is the product of all normal $p$-subgroups of $G, A_{m}$ and $S_{m}$ denote, respectively, the alternating and symmetric group on $m$ letters.

An automorphism group of a group is said to be regular if every non-trivial element of it is fixed-point-free.

LEMMA 1. If $R=\langle r\rangle$ is a regular automorphism group of order 3 of a finite group $H$ then, for every Abelian subgroup $A$ of $H,\left\langle A^{R}\right\rangle=\left\langle A, A^{r}\right\rangle$ and $\left\langle A, A^{r}\right\rangle$ is $A$ belian.

Proof: Let $H R$ be the natural semi-direct product of $H$ and $R$. Then $H R$ is a Frobenius group and hence $\left(h r^{-1}\right)^{3}=1$ for every element $h \in H$. Since $\left(h r^{-1}\right)^{3}=$ $h h^{r} h^{r^{2}}$, we have $h^{r^{2}}=\left(h^{-1}\right)^{r} h^{-1}$. Therefore $A^{r^{2}} \leqslant\left\langle A, A^{r}\right\rangle$. Let $a, b \in A$. Then $1=$ $a b(a b)^{r}(a b)^{r^{2}}=a b\left(a^{r} b^{r}\right) a^{r^{2}} b^{r^{2}}=a\left(b b^{r}\right)\left(a^{r} a^{r^{2}}\right) b^{r^{2}}=a\left(b^{-1}\right)^{r^{2}} a^{-1} b^{r^{2}}=\left[a^{-1}, b^{r^{2}}\right]$. Therefore $\left[a, b^{r^{2}}\right]=1$ and $\left[a^{r}, b\right]=1$. This means that $A^{r}$ centralises $A$. The lemma is proved.

An element of order 2 in a group is called an involution. The proof of the following well-known lemma is straightforward.

Lemma 2. Let $i, j$ be involutions. Then the following hold:

1. For every $x \in\langle i j\rangle, x^{i}=x^{j}=x^{-1}$.

2. If the order of $i j$ is finite and odd then $i, j$ are conjugate by an element in $\langle i j\rangle$ and by an involution in $i\langle i j\rangle$.

3. If the order of $i j$ is finite and even, then $\langle i j\rangle$ contains an involution which commutes with $i$ and $j$.

LEMMA 3. Suppose that $t$ is an involution in the automorphism group $A$ of a periodic group $G$. If $t$ is fixed-point-free then, for every $g \in G, g^{t}=g^{-1}, G$ is Abelian and $t$ lies in the centre of $A$. 
Proof: Let $H=G\langle t\rangle$ be the natural semi-direct product and $g \in G$. Since $C_{H}(t)=$ $\langle t\rangle$ and $t^{g} t=g^{-1} t g t=g^{-1} g^{t} \in G$, the order of $t^{g} t$ is odd, by part 3 of Lemma 2. By part 2 of Lemma 2 there exists an involution $i \in t G$ such that $t^{g i}=t$ and hence $g i=1$ or $g i=t$. If $g i=1$ then $g=i \in t G$ which is not the case. Thus $g i=t, g=t i$ and by part 1 of Lemma 2, $g^{t}=g^{-1}$. If $h \in G$ then $g h=\left(h^{-1} g^{-1}\right)^{-1}=\left(h^{t} g^{t}\right)^{t}=h g$, and $G$ is Abelian. If $a \in A$ then $g^{t a}=\left(g^{-1}\right)^{a}=\left(g^{a}\right)^{-1}=g^{a t}$ and hence $t a=a t$. The lemma is proved.

Lemma 4. Let $A$ be a proper subgroup of a group $H$. If $A$ contains no elements of order 3 and every element in $H \backslash A$ is a 3-element then $A$ is normal in $H$ and $A$ is nilpotent of class at most 2.

Proof: It is obvious that $A$ is normal in $H$. Let $x$ be an element of order 3 in $H$. Then, for every $a \in A, a x^{-1} \notin A$, so $\left(a x^{-1}\right)^{3}=1$ and hence $a a^{x} a^{x^{2}}=1$. It was proved by B.H. Neumann [7] that, in this situation, every element of $A$ commutes with each of its conjugates. By a result of Levi [4], $A$ is nilpotent of class at most 3 and the third term $T$ of the lower central series of $A$ is of exponent 3. By assumption, $T=1$. The lemma is proved.

Lemma 5. Let $G$ be a finite group with $\omega(G)=\{1,3,5\}$. Then $G$ contains a normal Sylow subgroup of prime index with non-cyclic centre.

Proof: Since the order of $G$ is divisible only by two distinct prime numbers, $G$ is soluble and $O_{p}(G) \neq 1$ for $p=3$ or $p=5$. If $F / O_{p}(G)$ is a minimal normal subgroup of $G / O_{p}(G)$ then $F$ is a Frobenius group and $Z\left(O_{p}(G)\right)$ is non-cyclic. By [3, Theorem V.8.15], $F / O_{p}(G)$ is cyclic and hence $F=G$.

Lemma 6. Let $\omega(G)=\{1,2,4,5\}$. If $V=O_{2}(G) \neq 1$ and $G=V D$ where $D$ is a dihedral group of order 10 generated by an involution $t$ and an element $r$ of order 5 then $V$ is elementary Abelian, $[V, t]=C_{V}(t)$ and $\left|V: C_{V}(t)\right|>2$.

Proof: Suppose that $V$ is elementary Abelian. If $v \in C_{V}(t)$ then $W=\left\langle v^{(r)}\right\rangle$ is a $D$-invariant subgroup of order $16, v \in[W, t]$ and $2<\left|W: C_{W}(t)\right| \leqslant\left|V: C_{V}(t)\right|$. Thus, it suffices to prove that $V$ is elementary Abelian. Since $G$ is locally finite, we can assume that $G$ is finite and proceed by induction on $|G|$. Suppose that $V$ is not elementary Abelian. Let $Z$ be a minimal normal subgroup of $G$. Then $Z \leqslant Z(V),|Z|=16$ and $V / Z$ is elementary Abelian. If $C / Z=C_{V / Z}(t)$ and $C$ contains an element $u$ of order 4 then $U=\left\langle u^{(r)}\right\rangle Z$ is a $D$-invariant subgroup and all elements in $U \backslash Z$ are of order 4 . But then $G$ contains an element of order 8. Thus $C$ is elementary Abelian. Let $v \in V$ be an element of order 4. Then, by induction, $V=\left\langle v^{\langle r\rangle}\right\rangle Z,|V|=2^{12}$ and $|C|=2^{8}$. If $c \in C \backslash Z$ then $U_{c}=\left\langle c^{(r)}\right\rangle Z$ is an elementary Abelian group of order $2^{8}$ and $U_{c_{1}} \cap U_{c_{2}}=Z$ if $c_{1} Z \neq c_{2} Z$. Thus $V=U_{c_{1}} U_{c_{2}}$ for some $c_{1}, c_{2}$ and there exists a uniquely defined $D$-homomorphism $\phi$ of the tensor product $X \otimes Y$ of a $D$-module $X=U_{c_{1}} / D$ and $D$-module $Y=U_{c_{2}} / Z$ into a $D$ module $Z$ which extends the map $x Z \otimes y Z \rightarrow[x, y]$. Let $F$ be a splitting field of $D$ over a 
field $F_{2}$ of order $2, \bar{X}=X \otimes_{F_{2}} F, \bar{Y}=Y \otimes_{F_{2}} F, \bar{Z}=Z \otimes_{F_{2}} F$. Then there exists a uniquely defined homomorphism $\bar{\phi}$ of $\bar{X} \otimes \bar{Y}$ into $\bar{Z}$ which extends $\phi$. Let $1 \neq \lambda \in F, \lambda^{5}=1$. We can choose bases $\left\{x_{i} \mid i=1, \ldots, 4\right\},\left\{y_{i} \mid i=1, \ldots, 4\right\}$ and $\left\{z_{i} \mid i=1, \ldots, 4\right\}$ of $\bar{X}, \bar{Y}, \bar{Z}$, respectively, such that $x_{i}^{r}=\lambda^{i} x_{i}, y_{i}^{r}=\lambda^{i} y_{i}, z_{i}^{r}=\lambda^{i} z_{i}, i=1, \ldots, 4$ and $x_{1}^{t}=x_{4}, x_{2}^{t}=x_{3}, y_{1}^{t}=y_{4}, y_{2}^{t}=y_{3}, z_{1}^{t}=z_{4}, z_{2}^{t}=z_{3}$. Denote $(x, y)=\bar{\phi}(x \otimes y)$. Since $\left(x_{i}, y_{i}\right)^{r}=\left(x_{i}^{r}, y_{i}^{r}\right)=\left(\lambda^{i} x_{i}, \lambda^{j} y_{j}\right)=\lambda^{i+j}\left(x_{i}, y_{j}\right)$, we see that

(1) $\left(x_{i}, y_{j}\right)=0$ for $(i, j) \in\{(1,4),(2,3),(3,2),(4,1)\}$, and, for other pairs $(i, j)$, $\left(x_{i}, x_{j}\right)=\alpha_{i j} z_{k}$ where $\alpha_{i j} \in F$ and $k$ is defined by $\lambda^{i+j}=\lambda^{k}, 1 \leqslant k \leqslant 4$.

Since $C$ is Abelian,

(2) $\left(x_{1}+x_{4}, y_{1}+y_{4}\right)=\left(x_{1}+x_{4}, y_{2}+y_{3}\right)=\left(x_{2}+x_{3}, y_{1}+y_{4}\right)$

$$
=\left(x_{2}+x_{3}, y_{2}+y_{3}\right)=0 \text {. }
$$

By (1), (2) gives $0=\left(x_{1}+x_{4}, y_{1}+y_{4}\right)=\left(x_{1}, y_{1}\right)+\left(x_{4}, y_{4}\right)=\alpha_{11} z_{2}+\alpha_{44} z_{3}$ and hence

$$
\left(x_{1}, y_{1}\right)=\left(x_{4}, y_{4}\right)=0 \text {. }
$$

Similarly,

$$
\begin{gathered}
\left(x_{1}, y_{2}\right)=\left(x_{4}, y_{2}\right)=\left(x_{1}, y_{3}\right)=\left(x_{4}, y_{3}\right)=0 \\
\left(x_{2}, y_{1}\right)=\left(x_{3}, y_{1}\right)=\left(x_{2}, y_{4}\right)=\left(x_{3}, y_{4}\right)=0 \\
\left(x_{2}, y_{2}\right)=\left(x_{3}, y_{3}\right)=0
\end{gathered}
$$

Thus $\bar{\phi}$ is the zero-homomorphism, $[x, y]=1$ for $x \in U_{c_{1}}, y \in U_{c_{2}}$ and $V$ is elementary Abelian. The lemma is proved.

LEMMA 7. Let $\omega(G) \subseteq\{1,2,4,5\}$. If $G$ is locally finite then either $G$ has a normal Sylow subgroup or $G=V D$ where $V=O_{2}(G)$ is a non-trivial elementary Abelian group and $D$ is a dihedral group of order 10.

Proof: Suppose first that $G$ is finite and proceed by induction on $G$. If $V=$ $O_{2}(G) \neq 1$ then, by induction, $G / V$ contains a normal Sylow 5-subgroup $P / V$. If $P=G$ then the conclusion is true. If $P \neq G$, then $P \neq V$ and, by [3, Theorem V.8.15], $|P: V|=5, G / V$ is a Frobenius group of order 10 or 20 . In particular, there exist involutions $x, y \in G \backslash P$ such that $x y$ is not a 2-element and hence $D=\langle x, y\rangle$ is a dihedral group of order 10. Let $H=O_{2}(G) D$. By Lemma $6, V=O_{2}(H)$ is elementary Abelian. If $G / V$ contains an element of order 4 then $G$ contains an element of order 8. Thus $H=G$ and the conclusion is true. If $O_{2}(G)=1$ then $P=O_{5}(G) \neq 1$ and $C_{G}(P) \leqslant P$. By Lemma 3, $Z(G / P)$ contains an element of order 2 and hence $G / P$ is a 2-group.

Suppose $G$ is infinite. If the product of every two 2-elements (every two 5-elements) in $G$ is a 2-element (respectively, 5-element) then a Sylow 2-subgroup (respectively, a Sylow 5-subgroup) of $G$ is normal in $G$. Suppose that there exist elements $x, y, z, t$ such that $x, y$ are 2-elements, $z, t$ are 5-elements, $x y$ is not a 2-element and $z t$ is not 
a 5-element. Then $H=\langle x, y, z, t\rangle$ is a finite group without non-trivial normal Sylow subgroups. Therefore, $V=O_{2}(H)$ is elementary Abelian and $H=V D$ where $D$ is a dihedral group of order 10. Let $C=C_{G}(V), N=C D$. By Lemma 6, every element in $C$ is of order 2. If $u \in G$ then $\langle u, H\rangle$ is finite and hence $H \leqslant C D=N$. Thus $N=G$ and the lemma is proved.

The following four lemmas can be verified by the coset enumeration algorithm (see, for instance [9]):

Lemma 8. Let $A=\langle a, b \mid R\rangle$. Table 1 gives the order of $A$ for some defining relations $R$.

Table 1

\begin{tabular}{l|r}
$R$ & $|A|$ \\
\hline$a^{3}, b^{3},(a b)^{3},\left(a b^{-1}\right)^{3}$ & \\
$a^{3}, b^{3},(a b)^{3},\left(a b^{-1}\right)^{5}$ & 27 \\
$a^{3}, b^{3},(a b)^{5},\left(a b^{-1}\right)^{5},\left(a b a^{-1} b\right)^{3}$ & 75 \\
$a^{3}, b^{3},(a b)^{5},\left(a b^{-1}\right)^{5},\left(a b a^{-1} b\right)^{5}$ & 62400
\end{tabular}

Table 2

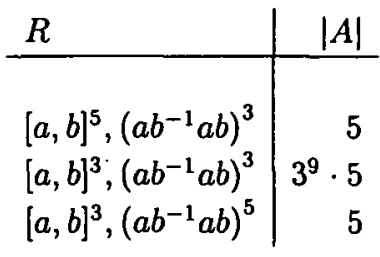

Lemma 9. Let $A=\left\langle a, b \mid a^{3}, b^{5},(a b)^{5},\left(a b^{-1}\right)^{5},\left(a b^{2}\right)^{5},\left(a b^{-2}\right)^{5}, R\right\rangle$. Table 2 gives the order of $A$ for various values of $R$.

Lemma 10. Let $A=\left\langle a, b, c \mid a^{3}, b^{3}, c^{3}, a b a^{-1} b^{-1},(a c)^{5},\left(a c^{-1}\right)^{3},(b c)^{3}, R\right\rangle$. Table 3 gives the order of $A$ for various values of $R$.

Lemma 11. Let $A=\left\langle a, b \mid a^{2}, b^{5}, R\right\rangle$. Table 4 gives the order of $A$ for various values of $R$.

\section{ProOfs of MaIN RESULTS}

Let $G$ be a group with $\omega(G)=\{1,3,5\}$. Note, that if $x^{3}=y^{3}=1$ for $x, y \in G$ then $(x y)^{3}=1$ or $\left(x^{-1} y\right)^{3}=1$. Indeed, $X=\langle x, y\rangle$ is finite by Lemma 8 and if $X \neq 1$ then, by Lemma $5, X$ contains a normal 5-subgroup $Y$ of index 3 , hence one of the elements $x y, x^{-1} y$ is not contained in $Y$. Since every element in $X \backslash Y$ is of order 3 , the assertion follows.

If $G$ contains a normal Sylow 5-subgroup $P$ then, by Lemma 4, $P$ is nilpotent of class at most 2 and hence $G$ is locally finite. By Lemma $5,|G / P|=3$. Suppose that there exist elements $a, b \in G$ of order 5 such that $a b$ is not a 5-element. Then the order of $c=a b$ is equal to 3 and $\langle a, b\rangle=\langle c, a\rangle$. If $\left(c a^{i}\right)^{3}=1$ for $i=1,2,3$ or 4 then, for $d=c a^{i}$, $\langle a, b\rangle=\langle c, a\rangle=\langle c, d\rangle$ is finite by Lemma 8. Suppose that, for $i=1,2,3,4,\left(c a^{i}\right)^{5}=1$. Since $\left(c^{-1} c^{a}\right)^{3}=1$ or $\left(c c^{a}\right)^{3}=1$, by Lemma $9,\langle a, b\rangle=\langle c, a\rangle$ is finite. By Lemma $5,\langle a, b\rangle$ contains a subgroup $T$ of order 9. Let $H$ be a maximal 3-subgroup of $G$ which contains $T$. Then $H$ is nilpotent of class at most 3 by [5]. Suppose that $H$ is not a normal 
subgroup of $G$. Then there exists an element $u \notin H$ of order 3 and an element $v \in H$ such that $v u$ is not a 3-element. Then the order of $v u$ is equal to 5 . Since $H$ is non-cyclic nilpotent, there exists an element $t \in H$ such that $\langle v, t\rangle$ is an elementary Abelian group of order 9. As above, $\langle t, u\rangle$ is finite and one of the elements $t u, t^{-1} u$ is of order 3. We can assume that $(t u)^{3}=1$. By Lemma $10,\langle u, v, t\rangle$ is finite which is impossible by Lemma 5 . Theorem 1 is proved.

Table 3

\begin{tabular}{l|rr|r}
$R$ & $|A|$ & $R$ & $|A|$ \\
\cline { 4 - 4 } & & & \\
$\left(b c^{2}\right)^{3},(a b c)^{3},\left(a b c^{2}\right)^{3},\left(a b^{2} c\right)^{3},\left(b a^{2} c\right)^{3}$ & 9 & $(a b)^{5},[a, b]^{5},(b a b)^{5}$ & 5 \\
$\left(b c^{2}\right)^{3},(a b c)^{3},\left(a b c^{2}\right)^{3},\left(a b^{2} c\right)^{3},\left(b a^{2} c\right)^{5}$ & 3 & $(a b)^{5},[a, b]^{4},(b a b)^{5}$ & $2^{9} \cdot 5$ \\
$\left(b c^{2}\right)^{3},(a b c)^{3},\left(a b c^{2}\right)^{3},\left(a b^{2} c\right)^{5},\left(b a^{2} c\right)^{3}$ & 3 & $(a b)^{5},[a, b]^{5},(b a b)^{4}$ & 360 \\
$\left(b c^{2}\right)^{3},(a b c)^{3},\left(a b c^{2}\right)^{5},\left(a b^{2} c\right)^{3},\left(b a^{2} c\right)^{3}$ & 3 & $(a b)^{5},[a, b]^{4},(b a b)^{4}$ & 1 \\
$\left(b c^{2}\right)^{3},(a b c)^{5},\left(a b c^{2}\right)^{3},\left(a b^{2} c\right)^{3},\left(b a^{2} c\right)^{3}$ & 3 & $(a b)^{4},[a, b]^{5},(b a b)^{5}$ & 1 \\
$\left(b c^{2}\right)^{3},(a b c)^{3},\left(a b c^{2}\right)^{5},\left(a b^{2} c\right)^{3},\left(b a^{2} c\right)^{5}$ & 1 & $(a b)^{4},[a, b]^{4},(b a b)^{5}$ & 360 \\
$\left(b c^{2}\right)^{3},(a b c)^{3},\left(a b c^{2}\right)^{5},\left(a b^{2} c\right)^{5},\left(b a^{2} c\right)^{3}$ & 1 & $(a b)^{4},[a, b]^{5},(b a b)^{4}$ & 160 \\
$\left(b c^{2}\right)^{3},(a b c)^{5},\left(a b c^{2}\right)^{3},\left(a b^{2} c\right)^{3},\left(b a^{2} c\right)^{5}$ & 1 & $(a b)^{4},[a, b]^{4},(b a b)^{4}$ & 2 \\
$\left(b c^{2}\right)^{3},(a b c)^{5},\left(a b c^{2}\right)^{3},\left(a b^{2} c\right)^{5},\left(b a^{2} c\right)^{3}$ & 75 & & \\
$\left(b c^{2}\right)^{5},(a b c)^{3},\left(a b c^{2}\right)^{3},\left(a b^{2} c\right)^{3},\left(b a^{2} c\right)^{3}$ & 3 & & \\
$\left(b c^{2}\right)^{5},(a b c)^{3},\left(a b c^{2}\right)^{3},\left(a b^{2} c\right)^{3},\left(b a^{2} c\right)^{5}$ & 75 & & \\
$\left(b c^{2}\right)^{5},(a b c)^{3},\left(a b c^{2}\right)^{3},\left(a b^{2} c\right)^{5},\left(b a^{2} c\right)^{3}$ & 1 & & \\
$\left(b c^{2}\right)^{5},(a b c)^{3},\left(a b c^{2}\right)^{5},\left(a b^{2} c\right)^{3},\left(b a^{2} c\right)^{3}$ & 1 & & \\
$\left(b c^{2}\right)^{5},(a b c)^{3},\left(a b c^{2}\right)^{5},\left(a b^{2} c\right)^{3},\left(b a^{2} c\right)^{5}$ & 1 & & \\
$\left(b c^{2}\right)^{5},(a b c)^{3},\left(a b c^{2}\right)^{5},\left(a b^{2} c\right)^{5},\left(b a^{2} c\right)^{3}$ & 1 & & \\
$\left(b c^{2}\right)^{5},(a b c)^{5},\left(a b c^{2}\right)^{3},\left(a b^{2} c\right)^{3},\left(b a^{2} c\right)^{3}$ & 1 & & \\
$\left(b c^{2}\right)^{5},(a b c)^{5},\left(a b c^{2}\right)^{3},\left(a b^{2} c\right)^{3},\left(b a^{2} c\right)^{5}$ & 1 & & \\
$\left(b c^{2}\right)^{5},(a b c)^{5},\left(a b c^{2}\right)^{3},\left(a b^{2} c\right)^{5},\left(b a^{2} c\right)^{3}$ & 1 & &
\end{tabular}

\section{Table 4}

Proof of Theorem 2: Let $G$ be a group with $\omega(G)=\{1,2,5\}$. By Lemma 11, every subgroup $H$ of $G$ generated by an element of order 5 and an element of order 2 is finite. If $H$ contains a normal Sylow 5-subgroup $P$ then, by Lemma 3, $P$ is elementary Abelian and hence $H$ is a dihedral group of order 10 . Suppose first that all subgroups of $G$ generated by an element of order 5 and an element of order 2 are of this type. Then the product of every two 5-elements is a 5-element and hence $O_{5}(G) \neq 1$. By Lemma 3 , $G / O_{5}(G)$ contains at most one involution and hence is a 2-group. Thus there exists an $H$ containing a non-trivial Sylow 2-subgroup $T$, and hence $G$ contains an elementary Abelian subgroup $V$ of order 4 . Let $F$ be the subgroup of $G$ generated by all involutions in $G$. If $F$ is a 2-group then the conclusion of the theorem is true.

Suppose that $F$ is not a 2-group. Then there exists an element $x \in G$ of order 5 such 
that $x=t_{1} t_{2} \cdots t_{s}$ where every $t_{i}, i=1,2, \ldots, s$ is an involution. Choose $x$ such that $s$ is minimal. Then $t_{1} \cdots t_{s-1}$ is an involution and $X=\left\langle x, t_{s}\right\rangle$ is a dihedral group of order 10 . Let $t=t_{s}$. If $C_{G}(t)$ contains only one involution then, by Lemma 2, every involution in $G$ is a conjugate of $t, t$ is contained in a subgroup which is a conjugate of $V$ and hence $C_{G}(t)$ contains an involution $u \neq t$. If $(u x)^{2}=1$ then the involution $u t$ centralises $x$ which is impossible by assumption. Thus $\langle u, x\rangle$ is a finite subgroup which has a non-trivial normal Sylow 2-subgroup. This subgroup is $t$-invariant and hence $X=\langle u, x, t\rangle$ is a finite group which has no non-trivial normal Sylow subgroups. It is easy to see that $H$ must contain an element of order 4 contrary to the assumption. Thus, $G$ contains a non-trivial normal Sylow $p$-subgroup $P$. If $p=5$ then, by Lemma $3, P$ is Abelian, $G / P$ contains only one involution and (i) holds. If $p=2$ then $P$ is elementary Abelian. Theorem 2 is proved. $\square$

Proof of TheOREM 3: Let $G$ be a group with $\omega(G)=\{1,2,4,5\}$.

LEMMA 12. Suppose that every finite non-trivial subgroup of $G$ contains a nontrivial normal Sylow subgroup. Then $G$ contains a non-trivial normal Sylow subgroup and (ii) or (iii) in the conclusion of Theorem 3 holds.

Proof: Let $F$ be the subgroup of $G$ generated by all involutions in $G$. If $F$ is a 2-group then $G / F$ does not contain an element of order 4 or 10 . By Theorem 3, the conclusion of the theorem is true. Hence $F$ is not a 2-group. It follows that there exists an element $x \in G$ of order 5 such that $x=t_{1} t_{2} \cdots t_{s}$ where every $t_{i}, i=1,2, \ldots, s$ is an involution. Choose $x$ such that $s$ is minimal. Then $t_{1} \cdots t_{s-1}$ is a non-trivial 2-element and, by Lemma 11, $X=\left\langle x, t_{s}\right\rangle$ is a finite group which cannot contain a normal Sylow 2-subgroup. Thus $X$ is a dihedral group of order 10 . Let $t=t_{s}$. If $C_{G}(t)$ contains only one involution then $C_{G}(t)$ is a finite 2-group, by [10], $G$ is locally finite and hence contains, by Lemma 7, a non-trivial normal Sylow 5-subgroup.

Suppose that $C_{G}(t)$ contains an involution $u \neq t$. If $(u x)^{2}=1$ then the involution $u t$ centralises $x$ which is impossible by assumption. Thus $\langle u, x\rangle$ has a normal Sylow 2-subgroup and hence $(u x)^{5}=1$. Thus $\langle u, x\rangle$ is a finite subgroup which has a nontrivial normal Sylow 2-subgroup. This subgroup is $t$-invariant and hence $\langle u, x, t\rangle$ is a finite group which has no non-trivial normal Sylow subgroups. This contradicts the assumption. Therefore, $G$ contains a non-trivial normal Sylow $p$-subgroup $P$. If $p=5$ then, by Lemma 3, $P$ is Abelian, $G / P$ contains only one involution and, by [3, Theorem V.8.15], (ii) holds. If $p=2$ then $P$ is locally finite. Let $x_{1}, \ldots, x_{7} \in P, y \in G \backslash P$. Then the order of $y$ is 5 and $Y=\left\langle x_{1}, \ldots, x_{7}, y\right\rangle$ is a finite group with a normal Sylow 2-subgroup $Z=P \cap Y$. By assumption, $\langle y\rangle$ acts regularly on $Z$ and $Z$ is nilpotent of class at most 6 by [2]. In particular, $\left[\left[\ldots\left[\left[x_{1}, x_{2}\right], x_{3}\right], \ldots\right], x_{7}\right]=1$. This means that $P$ is nilpotent of class at most 6 and (iii) holds. The lemma is proved.

Suppose that $G$ does not contain a non-trivial normal Sylow subgroup.

LEMMA 13. There exists a non-trivial elementary Abelian subgroup $V$ in $G$ such 
that $N=N_{G}(V)=V D$ where $D$ is generated by an element $r$ of order 5 and an involution $t$ with $r^{t}=r^{-1}$. Furthermore, if $V_{0}$ is a non-trivial normal subgroup of $N$ which is contained in $V$ then $N_{G}\left(V_{0}\right)=N$.

Proof: By Lemmas 7 and 12, there exists a finite subgroup $H$ of $G$ such that $U=O_{2}(H)$ is a non-trivial elementary Abelian group and $H=U D$ where $D$ is generated by an element $r$ of order 5 and an involution $t$ with $r^{t}=r^{-1}$. Let $V=C_{G}(U)$. Then $V$ is a locally finite 2-group and $N=V D$ is also locally finite. By Lemma $7, V$ is elementary Abelian and, since $U \leqslant V, C_{G}(V)=V$. Therefore $O_{2}\left(N_{G}(V)\right)=O_{2}\left(N_{G}(V)\right) D$ is also elementary Abelian and hence $O_{2}\left(N_{G}(V)\right)=V$. If $N_{G}(V) / V$ contains an invariant Sylow 5-subgroup then, by Lemma $12, N_{G}(V)$ is locally finite. By Lemma $7, N_{G}(V) / V \simeq D$.

If $N_{G}(V) / V$ does not contain an invariant Sylow 5-subgroup then, by Lemma 7, $N_{G}(V) / V$ contains a subgroup $S$ such that $O_{2}(S) \neq 1$ and $S / O_{2}(S) \simeq D$. The full preimage $U$ of $\mathrm{O}_{2}(S)$ in $G$ is again elementary Abelian and hence $U=V$ contrary to the choice of $S$. Thus $N_{G}(V)=N$.

Suppose that $1 \neq V_{0} \leqslant V$ and $V_{0}$ is normal in $N$. Then $V \leqslant C_{G}\left(V_{0}\right), C_{G}\left(V_{0}\right)$ is a $N$-invariant 2-group and $C_{G}\left(V_{0}\right) D$ is locally finite. Again, by Lemma 7, $C_{G}\left(V_{0}\right)$ is elementary Abelian and $C_{G}\left(V_{0}\right) \leqslant C_{G}(V)=V$. Thus $C_{G}(V)=V$ and $N_{G}\left(V_{0}\right) \leqslant$ $N_{G}\left(C_{G}\left(V_{0}\right)\right)=N_{G}(V)=N$. The lemma is proved.

Throughout the rest of the proof, $N, V, D, r, t$ are the subgroups and elements of $G$ defined in Lemma 13.

Lemma 14. If $v$ is an involution in $V$ then $C_{G}(v) \leqslant N$.

Proof: Suppose that there exists $x \in C_{G}(v) \backslash N$. Then $V_{0}=\left\langle v^{D}, x\right\rangle$ is a finite 2-subgroup in $C_{G}(v)$ and $V_{0} \nless N$. Let $V_{1}=V \cap V_{0}$. Then $V \leqslant C_{G}\left(V_{1}\right) \leqslant C_{G}\left(\left\langle v^{D}\right\rangle\right) \leqslant V$. If $V_{1}$ is normal in $V_{0}$ then $V_{0} \leqslant N_{G}\left(V_{1}\right) \leqslant N_{G}\left(C_{G}\left(V_{1}\right)\right)=N_{G}(V)=V$ contrary to the choice of $V_{0}$. Thus $V_{2}=N_{V_{0}}\left(V_{1}\right) \neq V_{3}=N_{V_{0}}\left(V_{2}\right)$. Let $y \in V_{3} \backslash V_{2}$. Then $V_{1}^{y} \neq V_{1}, V_{1}^{y} \neq$ $V, V_{1}^{y} \leqslant V_{2} \leqslant N$ and $\left|V_{1} V_{1}^{y}: V_{1}\right|=2$. But then $\left|V_{1}: C_{V_{1}}\left(V_{1}^{y}\right)\right|=2$, contradicting Lemma 6 .

Lemma 15. If $v$ is an involution in $N \backslash V$ then $C_{G}(v) \leqslant N$.

Proof: Since $\left\langle v, v^{r}\right\rangle / V \simeq D, v v^{r}$ is of order 5 and hence $v \in N_{N}(R)$ for some Sylow 5-subgroup $R$ of $N$. Therefore $v$ is a conjugate of $t$ in $N$ and we can assume that $v=t$. Let $V_{0}$ be a subgroup of order 4 in $C_{v}(t)$. Then $V_{1}=\left\langle V_{0}, t\right\rangle$ is an elementary Abelian group of order 8 in $C_{G}(t)$. Let $x$ be an element in $C_{G}(t) \backslash N$. Then $V_{2}=\left\langle V_{1}, x\right\rangle$ is a finite subgroup in $C_{G}(t)$. Let $V_{3}=V_{2} \cap N$. Then $V_{3}=V_{4} \times\langle t\rangle$ where $V_{4} \leqslant V$ and there exists $y \in N_{V_{2}}\left(V_{3}\right) \backslash N$. Since $\left|V_{3}: V_{4}\right|=2<\left|V_{4}\right|, C_{V_{4}}(y) \neq 1$. This contradicts Lemma 14 .

LEMMA $16 . \quad N=G$.

Proof: There exists an involution $v \in N$ such that $C_{N}(v)$ is non-Abelian. Since, By Lemma $15, C_{G}(t)$ is Abelian, not all involutions of $N$ are conjugate in $G$. If all 
involutions of $G$ are contained in $N$ then $N$ is normal in $G$ and hence $G \leqslant N_{G}(V)=N$. Suppose that $N \neq G$ and let $x$ be an involution in $G \backslash N$. Then there exists an involution $y \in N$ which is not a conjugate of $x$ in $G$. By Lemma 2, there exists an involution $z \in Z(\langle x, y\rangle)$. By Lemmas 14 and $15, z \in C_{G}(y) \leqslant N$ and $x \in C_{G}(z) \leqslant N$ contrary to the choice of $x$. The lemma and Theorem 3 are proved.

\section{REFERENCES}

[1] M. Hall Jr. 'Solution of the Burnside problem for exponent six', Mlinois J. Math. 2 (1958), 764-786.

[2] G. Higman, 'Groups and rings having automorphisms without non-trivial fixed elements', J. London Math. Soc. 32 (1957), 321-334.

[3] B. Huppert, Endliche Gruppen I (Springer Verlag, Berlin, Heidelberg, New York, 1979).

[4] F.W. Levi, 'Groups in which the commutator operation satisfies certain algebraical conditions', J. Indian Math. Soc. 6 (1942), 87-97.

[5] F. Levi, B.L. van der Waerden, 'Über eine besondere Klasse von Gruppen', Abh. Math. Sem. Univ. Hamburg 9 (1932), 154-158.

[6] B.H. Neumann, 'Groups whose elements have bounded orders', J. London Math. Soc. 12 (1937), 195-198.

[7] B.H. Neumann, 'Groups with automorphisms that leave only the neutral element fixed', Arch. Math. 7 (1956), 1-5.

[8] I.N. Sanov, 'Solution of Burnside's problem for exponent 4', (Russian), Leningrad State Univ. Annals [Uchenye Zapiski] Math. Ser. 10 (1940), 166-170.

[9] M. Schönert, et al., Groups, algorithms and programming (Lehrstuhl D für Mathematik, RWTH Aachen, 1993).

[10] V.P. Shunkov, 'On periodic groups with an almost regular involution', (Russian), Algebra $i$ Logika 11 (1972), 470-493. Algebra and Logic 11 (1972), 260-272.

[11] A.K. Zhurtov and V.D. Mazurov, 'A recognition of simple groups $L_{2}\left(2^{m}\right)$ in the class of all groups', (Russian), Sibirsk. Mat. Zh. 40 (1999), 75-78. Siberian Math. J. 40 (1999), 62-64.

Department of Mathematics

University of Manitoba

Winnipeg R3T 2N2

Canada

e-mail: ngupta@cc.umanitoba.ca
Institute of Mathematics

Novosibirsk 630090

Russia

e-mail: mazurov@math.nsc.ru 